\title{
EVALUATION OF RADIOLUCENCY AND SUBSIDENCE OF THE CEMENTLESS OXFORD MEDIAL UNICOMPARTMENTAL KNEE REPLACEMENT
}

\author{
Mamdouh Hefny, Jonathan Waite, Sherif Mostafa, Wael Sameer, \\ and Mahmoud El-Sebay.
}

\begin{abstract}
Department of Orthopaedic Surgery, Faculty of Medicine, Ain-Shams University, Cairo, Egypt.

Corresponding Author:

Mamdouh Hany Hefny.

E.mail:

Mamdouh.hefny@swft.nhs.uk.

Mobile: 00201003636004

Received: $18 / 3 / 2020$

Accepted: $16 / 4 / 2020$
\end{abstract}

Online ISSN: 2735-3540

\begin{abstract}
Background: The most popular unicompartmental knee replacement(UKR) prosthesis to treat medial compartment osteoarthritis (OA) is the Oxford knee (OUKR). This prosthesis now has cemented and cementless fixation options.The fixation of the new cementless implants has proved successful with significantly reduced radiolucent lines compared to the cemented prosthesis in the designer series. Unfortunately, some studies have reported tibial component subsidence as a specific complication to the cementless design.

Aim of the Work: This study aims to review the fixation and clinical outcomes of the cementless prosthesis. We report the incidence of radiolucent lines associated with both cementless and cemented tibial prosthesis. More importantly, to study the incidence of tibial component subsidence.
\end{abstract}

Materials and Methods: This is a prospective study of a consecutive series of the Oxford medial UKR. All patients had bone on bone arthritisfulfilling the criteria for anteromedial OA. All patients received cementless femur implants. The majority of patients received cementless tibial implants. Cemented tibial component was only used in elderly patients where the tibial metaphyseal bone was found potentially weak intraoperatively. Fluoroscopy-aligned radiographs were obtained post-operatively and at one-year.Radiolucent lines and subsidence were evaluated. All patients completed the Oxford knee questionnaire (OKS) at one-year follow-up.

Results: A total of 68 knees implanted in 59 patients completed the study. Fully cementless medial OUKR $(n=61)$, and cemented tibial with cementless femur $(n=7)$. The mean age was 67.5 years. Two patients required revision to TKR. The cemented design had higher incidence of radiolucent lines. One patient had Cementless tibial component subsidence. The median OKS was excellent in both groups.

Conclusion: Both designs of the OUKR are valid treatment optionswith excellent OKS. Radiolucent lines are less frequent with the cementless design buttibial component subsidence and fracture have a higher incidence compared to the cemented prosthesis.

Keywords: Medial, Unicompartmental knee replacement, cementless, subsidence.

\section{INTRODUCTION:}

Osteoarthritis (OA) of the knee joint is one of the commonest causes of chronic painful disability worldwide ${ }^{(1)}$. It affects different age groups in different ways. In the early stages, the disease is mostly affecting the medial compartment of the knee leaving the other compartments unaffected ${ }^{(2)}$. The goal of management of the disease is to provide a pain-free joint, reduce stiffness, and to minimize further damage to the joint. 


\section{Mamdouh Hefny, et al.,}

The modalities of treatment include physiotherapy, drug therapy, and surgery. Joint arthroplasty is spared for patients with end-stage arthritis not improving despite non-operative management ${ }^{(3)}$. Surgical treatment options include total knee replacement and partial knee replacement.

Early surgical techniques for knee arthroplasty, based on bony alignment, violated the soft tissue structures and used highly constrained prosthesis which resulted inhigh stresses at the bone cement interface leading to premature aseptic loosening and consequently its ultimate failure ${ }^{(4)}$.This led to the development of load sharing and less constrained implants which allow rotation and translation to occur in the joint, in an attempt to mimic the normal joint kinematics. In combination withthe preservation of the surrounding soft tissue structures improved joint stability, function and longevity have been achieved.

\section{Advances in knee arthroplastytechnique and implants have therefore greatly influenced the outcomes of the procedure.As the evolution of knee arthroplasty continues to pursue more joint salvaging techniques, i.e. less constrained implants and more soft tissue preservation, in order to maintain the normal biomechanics of the knee joint to achieve satisfactory patient outcomes and increased longevity.}

Uni-compartmental knee replacement (UKR) is the most joint conserving arthroplasty optionas it preserves all knee ligaments in an intact and functional state. It retains the normal rotational and translational motion of the joint. This allows more physiological joint biomechanics and hence, providesa more natural gait pattern with higher walking speed in comparison to total knee replacement (TKA). UKR has also been proven to offer a greater range of motion when compared to TKR. It is safer than TKR with lower morbidity and mortality. UKR has also been demonstrated to require a shorter hospital stay, and have lower costs. ${ }^{(5 \& 6)}$

The most popular UKR prosthesis to treat isolated medial compartment $\mathrm{OA}$ is the Oxford knee. The main design principle was the use of a fully-congruent mobile-bearing polyethylene insert to fulfill the function of the normal meniscus. This creates an increased surface area for load transmission, that has proven to have very low levels of wear ${ }^{(7)}$. The surgical technique allows for the accurate balance of medial soft tissues to their normal tension and subsequent correction of the coronal plane deformity. The prosthesis now has cemented and cementless fixation options. The cementless implant was designedto avoid errors of cementation and misinterpretation of radiolucent lines associated with the cemented version. The fixation of the new cementless implants has proved successful. The incidence of radiolucent lines was significantly reduced compared to the cemented prosthesis in a series published by one of the designing centres ${ }^{(8)}$. Unfortunately, some studies have reported tibial component subsidence as a specific complication to the cementless design ${ }^{(9,10)}$.

In our experience, this complication of the cementless design has almost exclusively seen in elderly patients with lower bone density. Having moved to use the cementless prosthesis for all patients, we now use a cemented tibial implant in elderly patients wherethe tibial metaphyseal boneis felt to be potentially weak intraoperatively.Younger patients with good bone quality stillreceive a cementless tibial component. Both groups receive a cementless femoral component since we have seen no complications related to that component and have not seen anyreported.

\section{AIM OF THE WORK:}

This study aims to review the fixation and clinical outcomes of the cementless 
prosthesis. We report the incidence of radiolucent lines associated with bothcementless and cemented tibial prosthesis. More importantly, to study the incidence of tibial component subsidence following the change of practice.

\section{MATERIAL AND METHODS:}

This is aprospective study of a consecutive series of the Oxford medial unicompartmental knee replacement in our institution. The vast majority of patients received cementless implants. Cemented tibial component was only used in elderly patients where the tibial metaphyseal bone was felt to be potentially weak intraoperatively.All patients had bone on bone arthritis isolated to the medial compartment of the knee with intact anterior cruciate ligament fulfilling the criteria for anteromedial OA (AMOA) ${ }^{(11)}$. All patients had pre-operative flexion deformity less than 15 degrees and flexion range of 110 degrees or more. All surgeries were performed at Warwick hospital or the Warwickshire Nuffield in the UK under one lead surgeon. All patientsreceived the Oxford Phase III Biomet UK Ltd, Swindon,UK. Surgery was performed through a minimally invasive medial arthrotomy using the microplasty instrumentation. All patients received two weeks of prophylactic anticoagulation of Apixaban $2.5 \mathrm{mg}$ twice daily. All patients received prophylactic antibiotics Teicoplanin \& Gentamicin on the induction of anaesthesia.Peri-operative complications were documented including intensive care unit (ICU) admission, deep vein thrombosis (DVT), pulmonary embolism (PE), blood transfusion, chest infection, and periprosthetic infection. All patients received the same postoperative protocol and completed a minimum of a one-year follow-up. Fluoroscopically-aligned radiographs were taken post-operatively and at a one-year follow-up. All patients completed the Oxford knee score questionnaire to evaluate their functional outcomes at one-year follow-up.

Fluoroscopy aligned radiographswere used, postoperative and at one year, to document the presence of radiolucent lines around the femoral and tibial components for both cemented and cementless prosthesis used. Radiolucent lines around the femoral component were evaluated on the lateral view radiographs. The tibial component was evaluated on AP radiographs and radiolucencies recorded by dividing the area under the tibial component into six zones as described in previous studies. Zone 7, the areaaround the tibial vertical wall was excluded from the evaluation in the cementless group as it is not coated with hydroxyapatite and therefore plays no role in implant fixation. ${ }^{(6)}$

Radiolucent lines for cemented and cementless prosthesis were classified into complete if all zones were involved or incomplete if not. Progressive radiolucency was reported if the radiolucencies were seen to increase in size or number of zones affected on subsequent radiographs. ${ }^{(12)}$

Subsidence was evaluated on the AP and lateral views and compared between post-operative and one-year films. Any subsidence of the tibial component was measured in distance from the prosthesis to the fibular head on the lateral radiographs. On the AP views the angle was measured between a line drawn parallel tothe flat undersurface of the tibial componentand the anatomical axis of the tibia.

The Oxford knee score questionnaire was used to evaluate the functional outcomes of patients at the end of the follow-up period.The score records the answer to 12 questions each scored 0 to 4 points creating a maximum score of 48/48. The scores were categorised into Excellent (range 41-48), Good(34-40), Fair (25-33) and Poor $(<24)$, as previously described in the literature ${ }^{(13)}$. 


\section{RESULTS:}

A total of 68 knees implanted in59 patients (nine bilateral) completed the study. Fully cementless medial OUKR was implanted in 61 knees, while cemented tibial and cementless femur were implanted in 7 knees. There was no loss to follow-up. No deaths were reported during the follow-up period.The mean age was 67.5 years at the time of surgery (range 41-86). The mean
BMI was 30.9 (range 20-43). Two patients required revisionto TKR. The first was revised for bearing dislocation due to an MCL traumatic rupture. The second was revised for deep infection.Radiographs at one year were obtained in allknees and were analysed for radiolucent lines. Oxford knee scores were completed for 61 knees at one year. The median OKS was 44 (Diagram 1). Excellent scores were achieved in $75 \%$ of the knees. None of the patients had poor scores.(Diagram2).

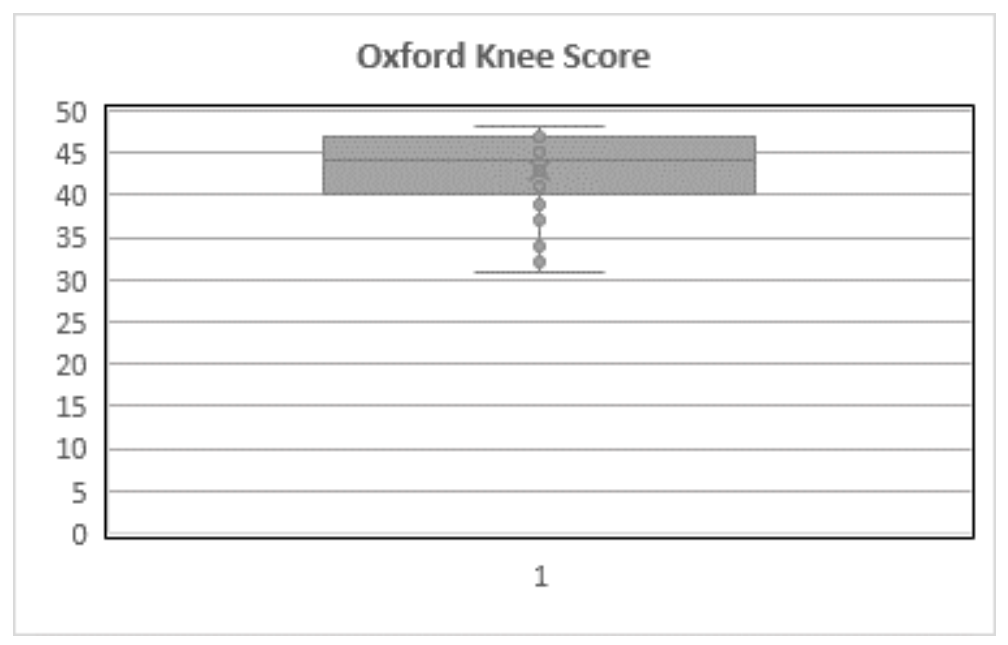

Diagram1. Full Cohort Oxford knee scores

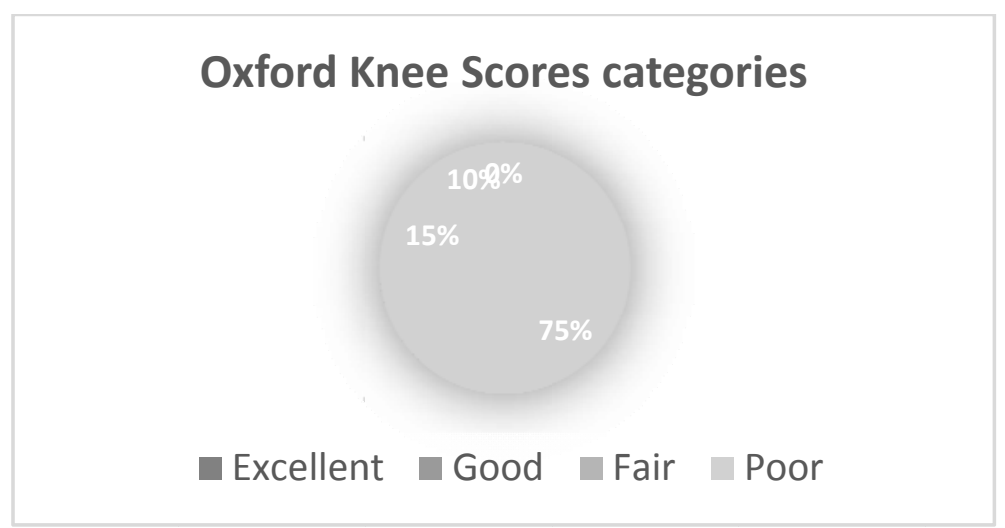

Diagram2. Oxford kneescores 


\section{Cementless tibial implant results}

For the 61 knees receiving a cementless tibial implant, the average age at the time of surgery was 67.2 years (range 41-82) with $29(47 \%)$ knees implanted in patients over 70 years at the time of surgery.

All patients completed Oxford score questionnaires pre-operatively and at a oneyear follow-up. The median postoperative Oxford score at one-year follow-up was44/48 (range 31-48). Excellent scores were achieved in $75 \%$ of cases.

\section{Radiolucent lines:}

There were no radiolucent lines associated with the femoral component.
There were no complete or progressive tibial radiolucencies. Incomplete tibial radiolucencies were seen in 25 knees $(40 \%)$ postoperatively but only 4 knees $(6.5 \%)$ at one year. These were all present on postoperative radiographs and were nonprogressive. Radiolucent lines were mostly in Zone 6. The location of radiolucent lines associated with the tibial component is shown in (Diagram 3).

Patients with postoperative radiolucent lines had median OKS of 44/48 (range 3348). This was the same for the few patients with radiolucent lines present at one year.

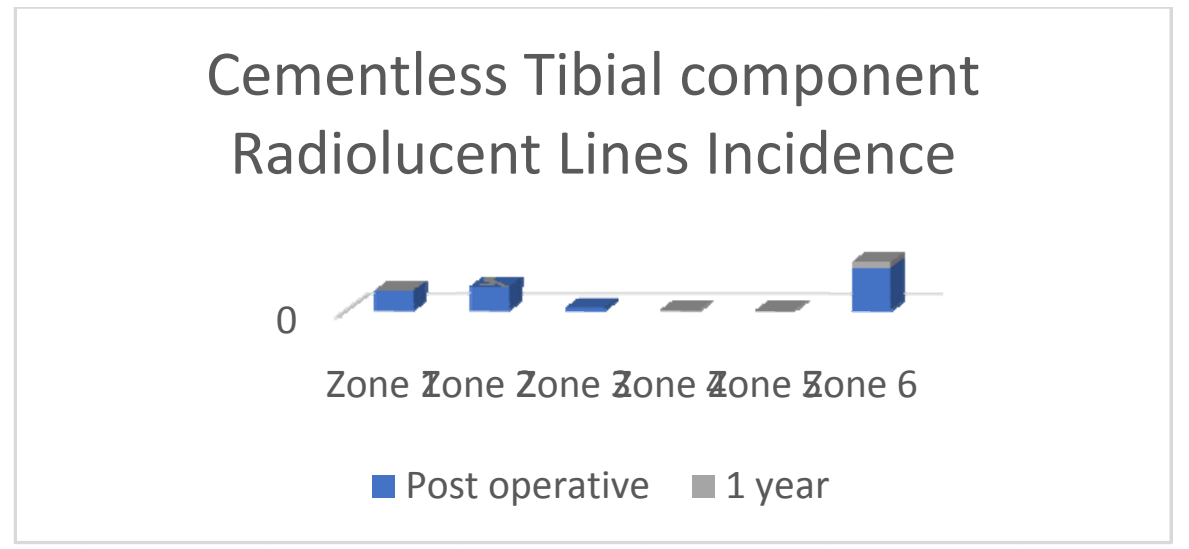

Diagram3. Incidence of tibial component radiolucent lines

\section{Subsidence:}

There was one tibial component subsidence identified in this patient cohort receiving cementless tibial prosthesis $(1.6 \%)$. This patient was a 67-year-old female. Subsidence occurred within the first two months with the tibial component angle measured on AP views changing to 94.7 degrees from 91.5 degrees on immediate postoperative radiographs. However, this had not progressed any on one-year radiographs. Posterior subsidence was not visualised on lateral view films. This patientreported increased pain at 8 weeks following surgery, however, this gradually settled and at one year her OKS was 44/48.

\section{Revision:}

Revision to TKR was required in two patients $(3 \%)$. One patient had a traumatic twisting knee injury during the early postoperative recovery period. He presented with posterior dislocation of the mobile bearing. Operative findings revealed a ruptured MCL, a laterally positioned vertical tibial cut compromising the ACL integrity. A decision was made to reconstruct the MCL and revise the implant to a TKR. The patient had a satisfactory outcome following revision. The second revision was at 10 months following primary surgery. The patient had a protracted course of conservative treatment after an initial 
reoperation following a fall causing complete wound dehiscence at 3/52 post-op. Despite antibiotic therapy revision to TKR was subsequently required for recurrent deep infection.

\section{Cemented tibial implant results:}

In sevenpatients in the cohort, an intraoperative decision was made to use a cemented tibial prosthesis due to poorproximal tibial bone stock. In each case, thiswas combined with a cementless femoral prosthesis. Their mean BMI was 28.5 (2037 ) and their mean age was 74 (range 58-
86). The male to female ratio was 2:5.Their median Oxford Knee score was 46/48. All had excellent scores.

\section{Radiolucent lines:}

There were no radiolucent lines associated with the femoral component. There were no complete or progressive tibial radiolucencies. There were no tibial radiolucencies seenpost-operatively but all 7 knees had partial radiolucencies at one year. These were all less than $2 \mathrm{~mm}$ in diameter with well-defined margins. (Diagram 4)

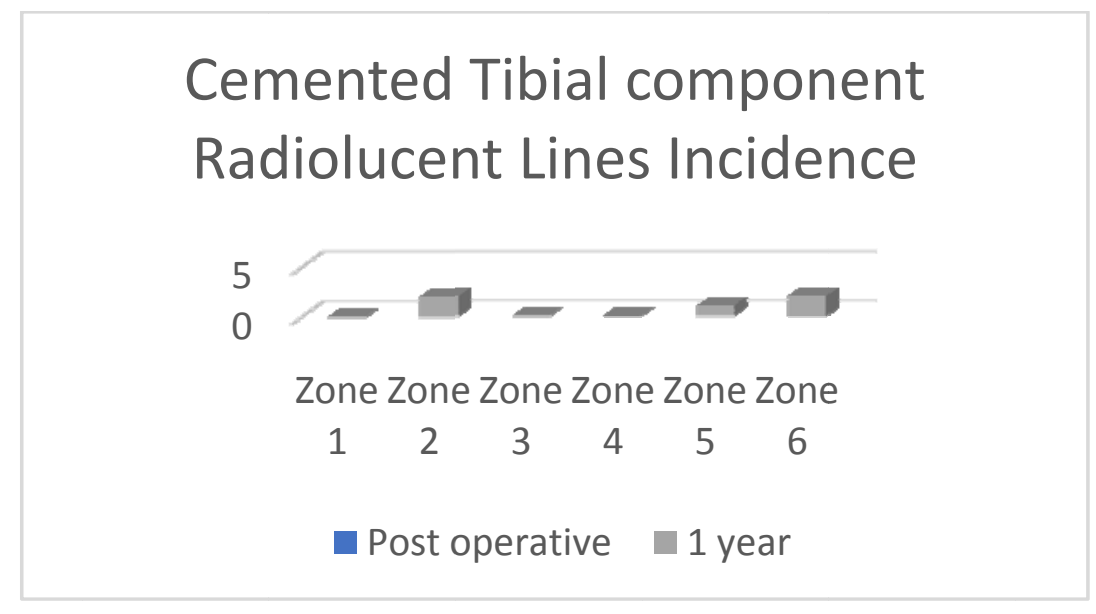

Diagram3. Cemented tibia radiolucent lines

\section{Subsidence:}

There was no tibial component subsidenceobserved in this group with a cemented tibial component.

\section{DISCUSSION:}

Our study shows similar findings to those few published reports of radiolucency under the tibial component of a UKR ${ }^{(5),(14)}$. We have used fluoroscopically-aligned radiographs to be confident that we are accurately recording radiolucencies as it has been shown that they are often missed on even well-aligned normal radiographs ${ }^{(12)}$.
The cemented OUKR tibial implant is known to have a higher incidence of radiolucent lines ${ }^{(6)}$. These usually develop during the first year, are well-defined, with a diameter less than $2 \mathrm{~mm}$, and have a sclerotic margin. They have been labelled physiologic by the Oxford group and are thought to be related to the unique loading pattern of the prosthesis. Several studies have reported good clinical outcomes despite the presence of these radiolucencies ${ }^{(12),(8,15)}$. This was the case in this series, in which the cemented tibial implant had incomplete radiolucent lines in all seven knees at one-year radiographs. However, all patients had excellent OKS 
However, outside the designer centre these radiolucencies are often a cause for concern. In cases where there is persistent proximal tibial pain post-operatively, it is difficult to be sure that the radiolucency does not represent aseptic loosening. Repeated use of fluoroscopically-aligned radiographs overtime should determine whether there is a progression of the radiolucency that would support loosening, but it is thought that decisions to revise the prosthesis, usually to a TKR, is often made without it. This is supported by the fact that the two most common causes of revision of OUKR in the NJR are pain and loosening ${ }^{(16)}$. The decision to manufacture cementless OUKRimplants was partly based upon this issue $^{(17)}$. There was a feeling that if radiolucency under the tibial prosthesis could be reduced, then it would lead to a reduction in the number of revisions and therefore better long-term implant survival. So far there have been very few studies to assess this outside of the designing centre.

Our data would appear to confirm previous findings. We have demonstrated that partial radiolucencies under the tibial prosthesis are common immediately postoperatively with the cementless component. This may represent incomplete seating of the component at the end of the procedure, or in some cases, just an image of the hydroxyapatite-bone interface before this has consolidated. By one year postoperatively almost all radiolucencies have been obliterated suggesting that the prosthesis gradually seats itself under loadbearing conditions and with the integration of bone to the hydroxyapatite coating ${ }^{(6),(17)}$. This is in contrast to the cemented prosthesis where solid fixation should be achieved immediately with curing of the bone cement intra-operatively. Hence radiolucencies are not present post-operatively. As the prosthesis is loaded during the first year it is common for radiolucencies to develop at the cement-bone interfaceas a consequence of the loading pattern, thermal necrosis, or due to development of a fibrous interface ${ }^{(15)}$.

It is hypothesized that this will lead tobetter long-term survival of the cementless prostheses as has been suggested by the New Zealand Joint Registry (NZJR) ${ }^{(18}$ ) which separates cemented and cementless UKRs, unlike the UK NJR.

The immediate stability of the cemented implant means that the incidence of tibial prosthesis subsidence is extremely low. However, this is not the same with the cementless component which is significantly higher ${ }^{(19)}$. The cementless design relies on the integrity of the proximal tibial bone for support. Resection of the subchondral bone plate to expose metaphyseal bone significantly weakens the area under the tibial prosthesis in the early post-operative phase. The bone under the prosthesis then goes through a period of consolidation as it is loaded once the patient begins weightbearing. A randomized control study using radio stereometric analysis demonstrated that the tibial component subsides by a mean of $0.23 \mathrm{~mm}$ in the first three months before the implant stabilizes ${ }^{(19)}$. There is therefore a period of time during which the bone is at risk of insufficiency failure leading to further subsidence of the prosthesis into the proximal tibia and at the extreme fracture.

The surgical technique demands accuracy in order to minimise this risk. Bone resection is kept to a minimum. The microplasty instrumentation was designed to make the depth of the tibial cut more reproducible such that $7 \mathrm{~mm}$ of bone is usually removed to allow implantation of a $3 \mathrm{~mm}$ thick tibial prosthesis and a $4 \mathrm{~mm}$ thick polyethylene bearing. In smaller knees when a small femoral component is used it is recommended to consider only a $6 \mathrm{~mm}$ resection and a $3 \mathrm{~mm}$ polyethylene bearing which is the thinnest available. The sagittal cut should be made to ensure there is no breach of the posterior tibial cortex and there is evidence that mild undermining of the 
intercondylar region with the horizontal cut will reduce the risk of fracture propagation distally. The sagittal tibial cut should be positioned as laterally as possible without violating the ACL footprint, such that it lies on the upslope of the medial tibial eminence, to allow implantation of the largest suitable tibial prosthesis. This should sit supported on the cortical rim of bone anteriorly, medially and posteriorly, rather than on the metaphyseal bone. If in between sizes it is recommended to use a prosthesis that slightly overhangs (ideally $<2 \mathrm{~mm}$ ) rather that one that underhangs. Despite surgeons adhering to the technique there have been published reports of tibial prosthesis subsidence ${ }^{(9 \& 10)}$, and fracture ${ }^{(8)}$ in greater numbers than with the cemented prosthesis. Our retrospective review of the first 5 years of using the cementless OUKR revealed a subsidence rate of $2.5 \%$ and one periprosthetic fracture. All the patients were $>70$ years old We have assumed that this was due to relatively weaker proximal tibial bone and now consider the use of a cemented tibial prosthesis if this is suspected intra-operatively. We have not seen any cases of subsidence or fracture when using the cemented prosthesis.

Our experience of subsidence is that it usually presents in the first 2 months postoperatively with an increase in proximal tibial pain after an initial period of pain reduction postoperatively. Critical use of fluoroscopically-aligned or well-aligned plain radiographs is mandatory in this situation to compare with immediate postoperative images. If there has been subsidence the treatment usually consists of a short period (4-6 weeks) of modified weight-bearing by which time the proximal tibial bone has consolidated and the patient can return to normal weight-bearing without the recurrence of pain. The subsidence rarely progresses beyond this point and good functional recovery is usually seen as with our case in this series (OKS 44/48 at one year). If there has been significant subsidence into valgus and flexion there may be instability within the joint, with a risk of bearing dislocation. If symptomatic, isolated tibial component revision to a cemented prosthesis (with the restoration of alignment) or revision to a TKR mayneed to be considered.

Periprosthetic proximal tibial fracture can usually be treated by reduction and fixation with reasonable long-term results. Occasionally conservative treatment is possible. If unsuccessful conversion to TKR is likely to be required.

Patients' factors may play an important role in the choice of the tibial prosthesis fixation method for this prosthesis. Elderly patients with poor tibial metaphysis bone quality due to periarticular osteoporosis, associated with reduced loading in the arthritic knee, may be at higher risk for tibial component subsidence. These patients may benefit more from receiving a cemented tibial implant. We accept the limitation of this study having a small sample size. Further multicenter randomized control trial may be required to confirm these findings and validate these findings.

\section{Conclusion:}

In conclusion, both cemented and cementless Oxford medial UKR are valid options for surgical treatment of patients with advanced medial compartment OA. Excellent patient functional outcomes are achievable with both implant designs. Persistent radiolucent lines are less frequent with the cementless design. However there has been no correlation between the presence of radiolucent lines and patientreported functional outcomes reported for either implant.

Tibial component subsidence and fracture are uncommon complications of the cementless OUKR, but have been recorded with a higher incidence than has been seen with the cemented prosthesis. However the risk of radiolucency with the cemented 
OUKR and the potential for that leading to a revision procedure needs to be balanced against this risk.Our current strategy is to use the cementless prosthesis in the vast majority of cases, but with occasional use of a cemented tibial component if we have concerns about the quality of the proximal tibial bone at the time of surgery.

\section{REFERENCES :}

1. Heidari B. Knee osteoarthritis prevalence, risk factors, pathogenesis and features: Part I. Caspian journal of internal medicine. 2011;2(2):205-12.

2. Vincent KR, Conrad BP, Fregly BJ, Vincent HK. The pathophysiology of osteoarthritis: a mechanical perspective on the knee joint. PM \& R : the journal of injury, function, and rehabilitation. 2012;4(5 Suppl):S3-S9.

3. Mora JC, Przkora R, Cruz-Almeida Y. Knee osteoarthritis: pathophysiology and current treatment modalities. Journal of pain research. 2018;11:2189-96.

4. Papas PV, Cushner FD, Scuderi GR. The History of Total Knee Arthroplasty. Techniques in Orthopaedics. 2018;33(1).

5. Blaney J, Harty H, Doran E, O'Brien S, Hill $\mathrm{J}$, Dobie I, et al. Five-year clinical and radiological outcomes in 257 consecutive cementless Oxford medial unicompartmental knee arthroplasties. Bone Joint J. 2017;99-b(5):623-31.

6. Hooper N, Snell D, Hooper G, Maxwell R, Frampton C. The five-year radiological results of the uncemented Oxford medial compartment knee arthroplasty. Bone Joint J. 2015;97-b(10):1358-63.

7. Barrett DS, Biswas SP, MacKenney RP. The Oxford knee replacement. A review from an independent centre. J Bone Joint Surg Br. 1990;72(5):775-8.

8. Campi S, Pandit HG, Dodd CAF, Murray DW. Cementless fixation in medial unicompartmental knee arthroplasty: a systematic review. Knee Surg Sports Traumatol Arthrosc. 2017;25(3):736-45.
9. Kamenaga T, Hiranaka T, Nakanishi Y, Takayama K, Kuroda R, Matsumoto $\mathrm{T}$. Valgus Subsidence of the Tibial Component Caused by Tibial Component Malpositioning in Cementless Oxford Mobile-Bearing Unicompartmental Knee Arthroplasty. J Arthroplasty. 2019; 34 (12):3 054-60.

10. Liddle AD, Pandit HG, Jenkins C, Lobenhoffer P, Jackson WF, Dodd CA, et al. Valgus subsidence of the tibial component in cementless Oxford unicompartmental knee replacement. Bone Joint J. 2014;96-b(3):345-9.

11. White SH, Ludkowski PF, Goodfellow JW. Anteromedial osteoarthritis of the knee. J Bone Joint Surg Br. 1991;73(4):582-6.

12. Gulati A, Chau R, Pandit HG, Gray H, Price AJ, Dodd CA, et al. The incidence of physio-logical radiolucency following Oxford unicompartmental knee replacement and its relationship to outcome. J Bone Joint Surg Br. 2009;91(7):896-902.

13. Murray DW, Fitzpatrick R, Rogers K, Pandit H, Beard DJ, Carr AJ, et al. The use of the Oxford hip and knee scores. The Journal of Bone and Joint Surgery British volume. 2007;89-B(8):1010-4.

14. Pandit HG, Campi S, Hamilton TW, Dada OD, Pollalis S, Jenkins C, et al. Five-year experience of cementless Oxford unicompartmental knee replacement. Knee Surg Sports Traumatol Arthrosc. 2017;25(3):694-702.

15. Tibrewal SB, Grant KA, Goodfellow JW. The radiolucent line beneath the tibial components of the Oxford meniscal knee. The Journal of Bone and Joint Surgery British volume. 1984;66-B(4):523-8.

16. National Joint Registry for England, Wales \& Northern Ireland. NJR. 16th Annual Report.

http://www.njrcentre.org.uk/njrcentre/News andEvents/NJR14thAnnualReportrecordnu mberofproceduresduring201617/tabid/1453/ Default.aspx2019.

17. Liddle AD, Pandit H, O'Brien S, Doran E, Penny ID, Hooper GJ, et al. Cementless fixation in Oxford unicompartmental knee replacement: a multicentre study of 1000 knees. Bone Joint J. 2013;95-b(2):181-7. 


\section{Mamdouh Hefny, et al.,}

18. New Zealand Orthopaedic Assocaition. New Zealand Joint Registry.Seventeen Year Report. 2016.

19. Kendrick BJ, Kaptein BL, Valstar ER, Gill HS, Jackson WF, Dodd CA, et al.
Cemented versus cementless Oxford unicompartmental knee arthroplasty using radiostereometric analysis: a randomised controlled trial. Bone Joint J. 2015;97-b (2): $185-91$ 
Evaluation of Radiolucency And Subsidence of the Cementless Oxford Medial Unicompartmental...

\author{
تقييم مفصل الركبة أو سفورد اللاأسنتي أحادي الجزء الإنسيابي من حيث شفافية الأشعة والهيوط \\ محمودالسباعي و وائل سمير وشريف مصطقى و ممدوح حفني و جوناثانويت

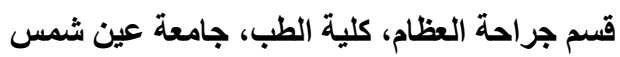

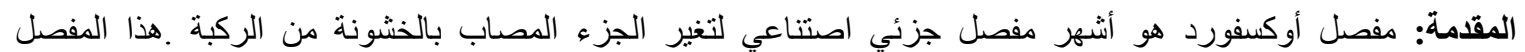

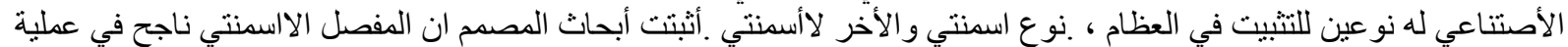

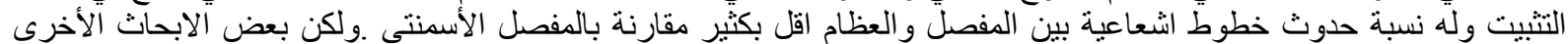
أنثارت الي حدوث هبوط في جزء المفصل الأسمنتي لعظمة الظنبوت.

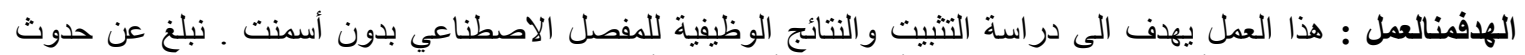

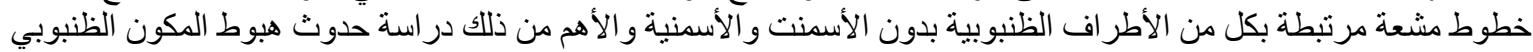

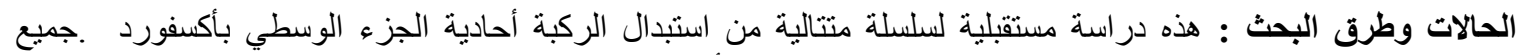

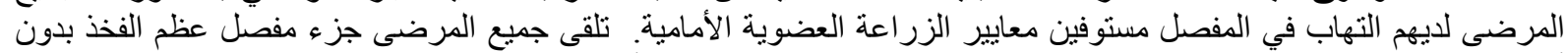

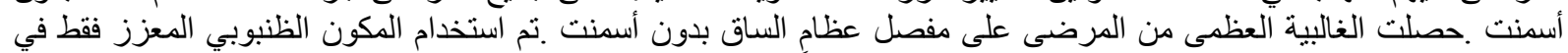

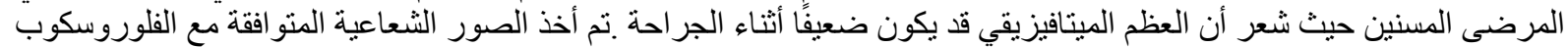

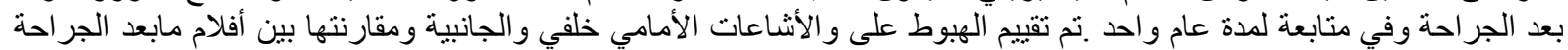

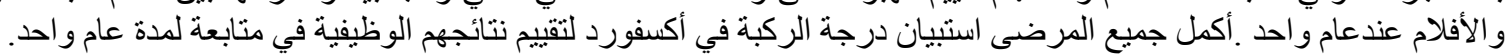

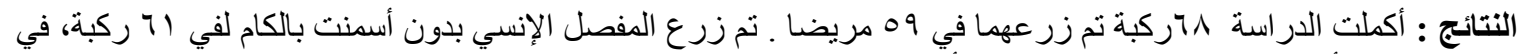

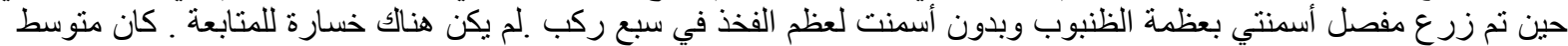

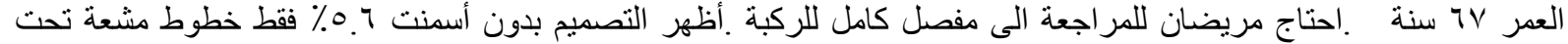

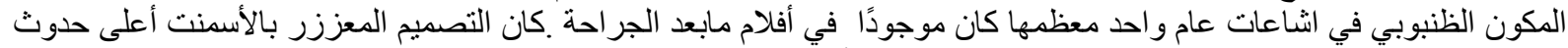

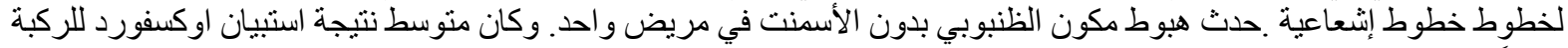
متازًا لكل الالمجمو عتين.

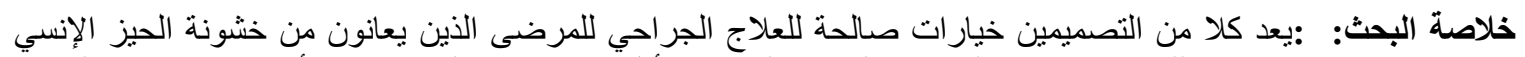

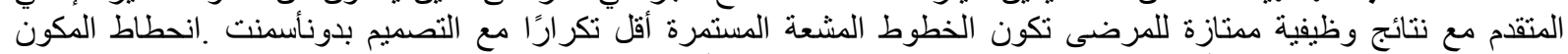

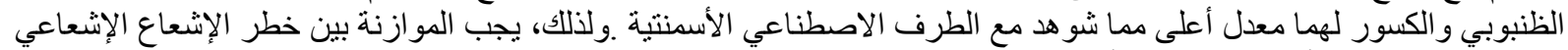

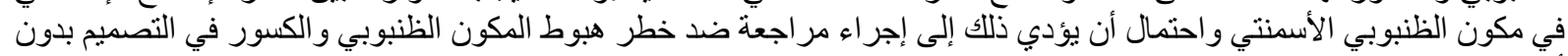

